\title{
Factors Associated with Utilization of Complete Postnatal Care Service in Baglung Municipality, Nepal
}

\author{
Sita Chhetri, ${ }^{1}$ Rajani Shah $\mathbb{D}^{2}$, and Laxmi Rajbanshi $^{3}$ \\ ${ }^{1}$ Charak Hospital Nursing College, Pokhara, Kaski, Nepal \\ ${ }^{2}$ Nepal Public Health Foundation, Kathmandu, Nepal \\ ${ }^{3}$ Teaching Hospital, Chitwan Medical College, Bharatpur, Nepal \\ Correspondence should be addressed to Rajani Shah; rajani_shah89@yahoo.com
}

Received 1 May 2020; Accepted 6 July 2020; Published 20 July 2020

Academic Editor: Robert Gaspar

Copyright (C) 2020 Sita Chhetri et al. This is an open access article distributed under the Creative Commons Attribution License, which permits unrestricted use, distribution, and reproduction in any medium, provided the original work is properly cited.

Background. Postnatal period is six weeks after birth. It is critical but is the most neglected period. A large proportion of maternal and neonatal deaths occur during 48 hours following childbirth. The utilization of the recommended three postnatal checkups within seven days after delivery, which plays a vital role in preventing maternal and neonatal deaths, is low in Nepal. Objective. This study is aimed at identifying the factors associated with the utilization of complete postnatal care (PNC) among mothers. Method. A cross-sectional study was carried out among 318 mothers in wards 1, 2, 3, and 4 of Baglung municipality, Nepal. Data was collected by semi-structured interviews. Descriptive analysis and comparison of characteristics of women/families with complete vs. partial postnatal checkups using multivariable logistic regression were done. Results. Among 314 respondents receiving at least one PNC, $78 \%$ had partial and $22 \%$ had complete PNC. Relatively advantaged caste/ethnicityBrahman/Chhetri ( $\mathrm{aOR}=3.18,95 \% \mathrm{CI}: 1.24-8.12)$ and Janajati $(\mathrm{aOR}=2.87,95 \% \mathrm{CI}: 1.09-7.53)$ - compared to Dalits, husbands working as a job holder in Nepal $(\mathrm{aOR}=3.49,95 \% \mathrm{CI}: 1.50-8.13)$, and delivery in a private hospital (aOR $=11.4,95 \% \mathrm{CI}: 5.40$ 24.2) were associated with having complete PNC. Conclusion. Although PNC attendance at least once was high, utilization of complete PNC was low. More focus to mothers from disadvantaged caste/ethnicity, those whose husbands are in foreign employment, and improvement in quality of care in government health facilities may increase the use of complete PNC.

\section{Introduction}

A large proportion of maternal and neonatal deaths occur during 48 hours following childbirth. Almost all (99\%) newborn and maternal deaths happen in developing countries. Globally, there were an estimated 303000 maternal deaths from complications related to pregnancy and childbirth in 2015 [1]. South Asia and Sub-Saharan Africa shared the highest proportion [2]. The first week of birth accounts for the highest number of the deaths [3]. In Nepal, maternal mortality ratio stands at 239 per 100000 live births, while neonatal mortality rate is 33 per 1000 live births [4]. Sustainable development goal aims at reducing the MMR to less than 70 per 100000 live births, reducing preventable deaths of newborn to less than $1 \%$, and providing postnatal care (PNC) for 90 percent of mothers by 2030 [5].
Postnatal period is up to six weeks after childbirth. The major purposes of PNC are to maintain and promote the health of the woman and her baby. It is generally the most neglected period in developing countries. Mothers and newborn babies do not receive PNC from a skilled health care provider during the first few days after delivery [6]. The national essential maternal and neonatal health care service package for Nepal recommends three PNC for all women, whether delivered at home or at health facility: first PNC within 24 hours, second and third ones on the third and seventh day, respectively [7].

Nepal Demographic and Health Survey 2016 showed that only $57 \%$ of mothers received a PNC within two days of delivery [4]. According to the Department of Health Services Nepal, the proportion of mothers having three PNC visits as per protocol stands at $11 \%$ in Baglung district, compared to 
$18 \%$ at the national level [8]. The national figure even declined to $16 \%$ in 2018 from 19\% in 2017. The low utilization of the service has been attributed to cultural and geographical factors as well as perceived low importance of care during the postpartum period [9]. Utilization of maternal health services is a complex phenomenon influenced by many factors. Sociodemographic factors such as age, education, wealth index, ethnicity, culture, decision making power, religion and service delivery environment are important determinants for the use of maternal health services $[10,11]$.

Although information on the use of PNC in Nepal is available [11-14], there is very little information about the use of the service according to protocol [9]. Hence, this study assessed the magnitude of and factors associated with the utilization of complete PNC service.

\section{Methods}

2.1. Study Area. Baglung district which represents a typical hilly district of Nepal and that has low utilization of PNC service was chosen [8]. It has population of 270000 . The center of the district, i.e., Baglung municipality, is about 275 kilometer west of Kathmandu, the capital of Nepal, and is connected to Nepal's one of the biggest cities, Pokhara. According to district population profile 2017, the total population of Baglung municipality is 63511 , with 1495 children of age 011 months [15]. Out of 14 wards of the municipality, ward numbers $1,2,3$, and 4 are city areas of the municipality and are connected to one government hospital and two private hospitals located at headquarter of the municipality by concrete road. It takes less than 30 minutes to reach the hospitals by walking from wards 1,2 , and 4 , whereas 10 to 60 minutes is needed from ward number 3 as it stretches from the market area to its outskirts.

2.2. Study Design and Population. A community based crosssectional study was conducted among mothers residing in the selected wards of the municipality who gave birth a week before data collection to the last 12 months.

2.3. Sample. Sample size of the study was calculated by using the population proportion formula taking into account the proportion of women using PNC service $(p)=50 \%[16,17]$, $95 \%$ confidence level, and margin of error $(d)=5.8 \%$.

Sample size $(n)=\frac{Z^{2} p q}{d^{2}}=\frac{(1.96 \times 1.96)(0.05) \times(0.05)}{(0.58)^{2}} 285.49$

Considering a non-response rate of $10 \%$, the final sample size of the study was 317.21 . Hence, a total of 318 respondents were included in the study.

2.4. Sampling. Baglung municipality, the only municipality of the district, was purposively selected as the study aimed at assessing the use of PNC service in an urban setting where the service is relatively more accessible. Four connected wards, namely, ward numbers 1 to 4 , were also purposively selected being the market area and its surrounding. All the mothers who had given birth one week prior to data collection for the past 12 months and residing in the selected wards of ward numbers 1,2, and 3 and some mothers of ward number 4 were interviewed by house screening.

2.5. Data Collection and Procedure. Data was collected from $25^{\text {th }}$ June to $25^{\text {th }}$ July 2017 by face-to-face interview using a pretested [18] semi-structured questionnaire in Nepali language. The questionnaire included questions on use of PNC service, socio-demographic variables (age, education and occupation of respondents, caste/ethnicity, annual family income, and occupation of husbands), obstetrical variables (parity, antenatal care (ANC), place of delivery, duration of hospital stay, and complication during delivery), and awareness of PNC service. The number of mothers was identified from the registry of female community health volunteers of the respective wards and immunization registry of the local health facility. Then, house-to-house visit was done for data collection. Data was collected by the first author. The collected data was edited on the same day throughout the data collection period.

\subsection{Variables}

2.6.1. Dependent Variable. The dependent variable was the 'utilization of complete PNC.'

2.6.2. Independent Variables. Independent variables include socio-demographic characteristics, ANC visit, type of facility used for childbirth, complication during childbirth, duration of hospital stay, and awareness of PNC services.

\subsection{Operational Definition}

2.7.1. Utilization of Complete PNC. Utilization of complete PNC refers to at least three PNC within seven days after delivery. It was classified into two categories based on frequency and timing of postnatal checkup: 'partial' means one and/or two PNC within 24 hours and on third day following delivery, respectively, while 'complete' means three PNC with at least the third one on the seventh day following delivery.

(1) Caste/Ethnicity. 'Brahman/Chhetri' (so-called upper caste group), 'Janajati', and 'Dalits' [19]

(2) Education. Education was measured in terms of the ability to read and write and level of education completed. The four categories included 'no schooling,' 'basic education' (completion of any of the grades from 1 to 8 ), 'secondary education' (completion of any of 9 to 12 grades), and 'higher education' (bachelor's degree or higher) [20]

(3) Economic Status. Total yearly income of the household in Nepalese rupee (NPR) was classified into three categories: 'less than 250000,' '250000-600000,' and 'more than 600000' [21, 22]

(4) Parity. The parity of the recent birth was categorized as 'one' and 'two or more' for further analysis. Type 
of Family. The type of family was categorized as 'nuclear' and 'joint'

(5) Number of ANC Visit. The number of ANC visit a respondent had during her last pregnancy was counted. The responses were grouped as 'no visit,' 'less than 4 visits,' and ' 4 or more visits'

(6) Childbirth Complication. Complication that arose during last childbirth. It was categorized as 'yes' and 'no'

(7) Type of Health Facility Used for Childbirth. 'Government facility' and 'private facility'

(8) Duration of Hospital Stay after Delivery. The duration of hospital stay after delivery was categorized as ' 6 hours or less,' ' 7 to 24 hours,' and 'more than 24 hours' $[3,7,23]$.

(9) Awareness of PNC Services. It refers to information regarding meaning of postnatal period, timing and frequency of PNC visit, recommended health care provider for PNC, types of PNC services, and danger signs that may arise during postnatal period. It was classified into two categories based on the mean score: 'good awareness' (mean $\geq 5.50)$ and 'poor awareness' (mean < 5.50)

2.8. Data Processing and Analysis. Collected data were checked daily for completeness, consistency, and accuracy. The data were coded and then entered in data entry software EpiData 3.1 [24] and analyzed in the software Statistical Package for the Social Sciences version 18 [25]. Descriptive and inferential statistics were used for data analysis. As descriptive statistics, frequency, percentage, mean and standard deviation were used. In inferential statistics, chi-square test was applied to find out the association between level of utilization of PNC and independent variables. The variables that showed significant association at $5 \%$ level of significance were further analyzed to estimate unadjusted and then adjusted odds ratio (aOR) using multivariable logistic regression [26] to determine the factors associated with the utilization of complete PNC and their strength of association.

2.9. Ethics and Consent to Participate. Ethical approval was obtained from Chitwan Medical College-Institutional Review Committee. Permission to carry out the study was taken from municipality office of Baglung. Verbal informed consent was obtained from each respondent by clarifying the purpose of the study prior to the data collection. Confidentiality was maintained by not disclosing the information to anyone else and using it for research purpose only. Respondents' dignity was maintained by giving the right to reject or discontinue from the study at any time without any penalty.

\section{Results}

3.1. Sociodemographic Characteristics of the Respondents. Among 318 total respondents, 39\% were in the age group 20-24 years. Regarding caste/ethnicity, about $47 \%$ were Brahman/Chhetri. Around $91 \%$ of the respondents belonged to the Hindu by religion. About two-thirds (63.7\%) of the respondents had secondary level schooling. With regard to occupation, more than half (55.2\%) of the respondents were involved in household work. Regarding the type of family, $54 \%$ of the respondents belonged to a nuclear family. More than one-third (35.6\%) of the respondents stated that the annual income of their family was 250000 to 600000 NPR. About $31 \%$ of the husbands were involved in foreign employment. Nearly half (48.7\%) of the respondents had given birth for the first time (Table 1).

3.2. Obstetrical Characteristics of the Respondents. Nearly all (99.4\%) of the respondents had ANC checkup during last pregnancy. Of these, around $88 \%$ had four or more ANC. Regarding the place of delivery, almost all (98.7\%) of the respondents had their last delivery in a health facility, while $84 \%$ of these used government hospitals for delivery. Around three-quarters $(74.8 \%)$ of the mothers delivered by spontaneous vaginal delivery, while about $22 \%$ of the respondents had caesarean section. Slightly more than two-thirds (67.2\%) of the births were assisted by a staff nurse/auxiliary nurse midwife (ANM). Regarding duration of hospital stay after delivery, $50 \%$ of the respondents stayed for seven to 24 hours. About $19 \%$ of the mothers had complication during the last childbirth. Half of the respondents (50.6\%) had poor level of awareness on PNC services (Table 2).

3.3. Utilization of PNC. Of the total respondents, almost all (98.7\%) had PNC. Among them, $78 \%$ had partial utilization (1-2 visit), while $22 \%$ had complete PNC. About $62 \%$ of the respondents received the PNC from nurses, and $85 \%$ of the respondents received the service from government hospital (Table 3).

3.4. Factors Associated with Utilization of Complete PNC. Caste/ethnicity, occupation of respondent's husband, type of health facility used for delivery, complication during delivery, duration of hospital stay after delivery, and awareness level on PNC service were found to be significantly associated with the utilization of complete PNC (Table 4).

3.5. Odds Ratio of Factors Associated with Use of Complete $P N C$. In multivariable logistic regression analysis, only caste/ethnicity, occupation of respondent's husband, and type of health facility where delivery happened were significantly associated with the utilization of complete PNC. The mothers who belonged to Brahman/Chhetri ( $\mathrm{aOR}=3.18,95 \% \mathrm{CI}$ : 1.24-8.12) and Janajati ( $\mathrm{aOR}=2.87,95 \%$ CI: 1.09-7.53) caste/ethnicity were more likely to have complete PNC as compared to Dalits. Similarly, respondents whose husbands were job holders within the country were more likely $(\mathrm{aOR}=3.49$, 95\% CI: $1.50-8.13)$ to get complete PNC as compared to those whose husbands were in foreign employment. Respondents who delivered their last child at a private hospital had higher likelihood (aOR=11.4, 95\% CI: 5.40-24.2) of having complete PNC compared to those who delivered in a government hospital (Table 5). 
TABLE 1: Sociodemographic characteristics of respondents $(n=318)$.

\begin{tabular}{|c|c|c|}
\hline Variables & Frequency & Percentage \\
\hline \multicolumn{3}{|l|}{ Age of mothers (in years) } \\
\hline Less than 20 & 26 & 8.2 \\
\hline 20 to 24 & 124 & 39.0 \\
\hline $25-29$ & 120 & 37.7 \\
\hline 30 and above & 48 & 15.1 \\
\hline \multicolumn{3}{|l|}{ Ethnicity } \\
\hline Brahman/Chhetri & 148 & 46.5 \\
\hline Janajati & 95 & 29.9 \\
\hline Dalits & 75 & 23.6 \\
\hline \multicolumn{3}{|l|}{ Religion } \\
\hline Hindu & 290 & 91.2 \\
\hline Non-Hindu* & 28 & 8.8 \\
\hline \multicolumn{3}{|l|}{ Maternal education } \\
\hline No schooling & 13 & 4.1 \\
\hline Basic education & 49 & 15.4 \\
\hline Secondary education & 189 & 59.4 \\
\hline Higher education & 67 & 21.1 \\
\hline \multicolumn{3}{|l|}{ Maternal occupation } \\
\hline Household work & 167 & 52.5 \\
\hline Farmer & 55 & 17.3 \\
\hline Service & 48 & 15.1 \\
\hline Business & 34 & 10.7 \\
\hline Labour & 14 & 4.4 \\
\hline \multicolumn{3}{|l|}{ Type of family } \\
\hline Nuclear & 173 & 54.4 \\
\hline Joint & 145 & 45.6 \\
\hline \multicolumn{3}{|c|}{ Annual family income (in NPR) } \\
\hline Less than 250000 & 120 & 37.7 \\
\hline 250000 to 600000 & 113 & 35.6 \\
\hline More than 600000 & 85 & 26.7 \\
\hline \multicolumn{3}{|c|}{ Occupation of respondent's husband } \\
\hline Foreign employment & 98 & 30.8 \\
\hline Business & 83 & 26.1 \\
\hline Service (job holder) & 81 & 25.5 \\
\hline Labour & 56 & 17.6 \\
\hline \multicolumn{3}{|l|}{ Parity } \\
\hline One & 155 & 48.7 \\
\hline Two or more & 163 & 51.25 \\
\hline
\end{tabular}

*Buddhist, Christian, and Islam.

\section{Discussion}

The study showed that about $99 \%$ of the respondents had utilized PNC service at least once, $34.7 \%$ had received two PNC, and only $21.7 \%$ of them had received the service three times within seven days following delivery. A similar study carried out in India found that $36.6 \%$ of the respondents had received one postnatal checkup, $18.5 \%$ received two, and $14.7 \%$ had three postnatal checkups [27]. Likewise, a study conducted in Ethiopia revealed that $57.4 \%$ of the mothers
TABLE 2: Obstetrical characteristics of respondents $(n=318)$.

\begin{tabular}{|c|c|c|}
\hline Variables & Frequency & Percentage \\
\hline \multicolumn{3}{|l|}{ Had ANC during last pregnancy } \\
\hline Yes & 316 & 99.4 \\
\hline No & 2 & 0.6 \\
\hline \multicolumn{3}{|l|}{ Number of ANC visit $(n=316)$} \\
\hline$<4$ times & 37 & 11.7 \\
\hline$\geq 4$ times & 279 & 88.3 \\
\hline \multicolumn{3}{|l|}{ Place of delivery of last child } \\
\hline At health facility & 314 & 98.7 \\
\hline At home & 4 & 1.3 \\
\hline \multicolumn{3}{|c|}{$\begin{array}{l}\text { Type of health facility used for delivery } \\
(n=314)\end{array}$} \\
\hline Government hospital & 265 & 84.4 \\
\hline Private hospital & 49 & 15.6 \\
\hline \multicolumn{3}{|l|}{ Type of delivery } \\
\hline Spontaneous vaginal delivery & 238 & 74.8 \\
\hline Assisted vaginal delivery & 11 & 3.5 \\
\hline Caesarean section & 69 & 21.7 \\
\hline \multicolumn{3}{|l|}{ Birth attendant at hospital } \\
\hline Doctor & 103 & 32.8 \\
\hline Staff nurse/ANM & 211 & 67.2 \\
\hline \multicolumn{3}{|l|}{ Birth attendant at home $(n=4)$} \\
\hline Relative/friend & 2 & 50.0 \\
\hline Staff nurse & 1 & 25.0 \\
\hline No one & 1 & 25.0 \\
\hline \multicolumn{3}{|c|}{$\begin{array}{l}\text { Duration of hospital stay after delivery } \\
(n=314)\end{array}$} \\
\hline$\leq 6 \mathrm{hrs}$ & 65 & 20.7 \\
\hline $7-24$ hrs. & 157 & 50.0 \\
\hline$>24$ hrs. & 92 & 29.3 \\
\hline \multicolumn{3}{|l|}{ Had complication during childbirth } \\
\hline Yes & 60 & 18.9 \\
\hline No & 258 & 81.1 \\
\hline \multicolumn{3}{|c|}{ Type of the complications** $(n=60)$} \\
\hline Prolonged labour & 29 & 48.4 \\
\hline Severe vaginal bleeding & 18 & 30.0 \\
\hline Meconium stained liquor & 12 & 20.0 \\
\hline Retained placenta & 5 & 8.3 \\
\hline Raised blood pressure & 5 & 8.3 \\
\hline \multicolumn{3}{|l|}{ Level of awareness on PNC services } \\
\hline Poor awareness $(<5.50)$ & 161 & 50.6 \\
\hline Good awareness $(\geq 5.50)$ & 157 & 49.4 \\
\hline
\end{tabular}

${ }^{* *}$ Multiple responses.

had one PNC, $40.4 \%$ had two PNC, and $2.2 \%$ had three or more PNC [28]. The difference in the proportions in the present and the previous studies may be attributed to the study setting and study population. Regarding the level of the utilization of PNC, $78 \%$ of the respondents had partial (1 to 2 times) utilization, while only $22 \%$ of the respondents had utilized complete PNC. A national figure showed that 
TABLE 3: Utilization of PNC.

\begin{tabular}{lcc}
\hline Variables & Frequency & Percentage \\
\hline Received PNC $(n=318)$ & 314 & \\
$\quad$ Yes & 4 & 1.3 \\
No & & \\
Frequency of PNC $(n=314)$ & 205 & 65.3 \\
$\quad$ Once & 40 & 12.7 \\
Two times & 69 & 22.0 \\
Three times & & \\
Level of utilization of PNC & 245 & 78.0 \\
$\quad$ Partial & 69 & 22.0 \\
Complete & & \\
PNC provided by & 108 & 34.3 \\
$\quad$ Doctors & 194 & 61.9 \\
Staff nurse/ANM & 12 & 3.8 \\
Health assistant & & \\
Place for PNC & 268 & 14.6 \\
$\quad$ Government hospital & 46 & \\
Private hospital/clinic & &
\end{tabular}

in Nepal $19 \%$ of women had PNC checkup as per protocol [9]. Similarly, a study conducted in Myanmar demonstrated that the prevalence of the utilization of complete PNC was $25.2 \%$ [29].

In the present study, respondents who belonged to Brah$\mathrm{man} / \mathrm{Chhetri}(\mathrm{aOR}=4.33,95 \% \mathrm{CI}: 1.59-11.80)$ and Janajati $(\mathrm{aOR}=4.06,95 \% \mathrm{CI}: 1.44-11.4)$ caste/ethnicity were more likely to have complete PNC as compared to Dalits. Consistently, a study conducted in Nepal showed that the Tamang ethnic group was less likely to have had PNC than Brahman/Chhetri (aOR $=0.15,95 \%$ CI: 0.05-0.44) [11]. Similarly, another study in Nepal revealed that women from Janajati were less likely to utilize the maternal services $(\mathrm{aOR}=0.75)$ than women from Brahman/Chhetri [12]. A systematic review and meta-analysis on inequities in the utilization of PNC in the low- and middle-income countries concluded that the use of PNC varies significantly by socioeconomic status of people [30]. The differences in the service utilization might be explained by the differentials in socioeconomic status reflected in the hierarchy of caste/ethnicity persistent in Nepal [31].

The respondents whose husbands were job holders in Nepal were more likely $(\mathrm{aOR}=3.6,95 \% \mathrm{CI}: 1.54-8.66)$ to have complete PNC as compared to the respondents whose husbands were in foreign employment. Another study in Nepal revealed that mothers whose husbands were involved in the technical field (aOR $=1.7,95 \%$ CI: 1.35-2.17) and labour $(\mathrm{aOR}=1.3,95 \% \mathrm{CI}: 1.10-1.77)$ were more likely to receive PNC than the respondents whose husbands were involved in agriculture [14]. Another study supports the findings of the current study that women whose husbands were employees of the government or private sectors $(\mathrm{aOR}=2.60$, $95 \% \mathrm{CI}=1.21-5.98$ ) tended to be more likely to use PNC service than women whose husbands were involved in agriculture and/or labour work [32]. In the current study, however, the
TABLE 4: Factors associated with the utilization of complete PNC.

\begin{tabular}{|c|c|c|c|}
\hline Variables & $\begin{array}{l}\text { Utilization of } \\
\text { Fully utilized, } \\
\text { N. (\%) }\end{array}$ & $\begin{array}{l}\text { f PNC services } \\
\text { Partial utilized, } \\
\text { N. (\%) }\end{array}$ & $p$ value \\
\hline \multicolumn{4}{|l|}{ Age groups (in years) } \\
\hline Less than 30 & $58(21.8)$ & $270(78.2)$ & 0.846 \\
\hline 30 and above & $11(22.1)$ & $37(77.1)$ & \\
\hline \multicolumn{4}{|l|}{ Ethnicity } \\
\hline Janajati & $23(24.7)$ & $70(75.3)$ & 0.029 \\
\hline Brahman/Chhetri & $38(32.3)$ & $109(74.1)$ & \\
\hline Dalits & $8(10.8)$ & $66(89.2)$ & \\
\hline \multicolumn{4}{|l|}{ Education status } \\
\hline No schooling & $3(23)$ & $10(77)$ & \\
\hline Basic education & $8(17)$ & $38(83)$ & 0.668 \\
\hline Secondary education & $40(21)$ & $148(79)$ & \\
\hline Higher education & $18(27)$ & $49(73)$ & \\
\hline \multicolumn{4}{|l|}{$\begin{array}{l}\text { Occupation of } \\
\text { respondents }\end{array}$} \\
\hline Household work & $38(23.0)$ & $127(77.0)$ & \\
\hline Farmer & $10(18.9)$ & $43(81.1)$ & \\
\hline Service & $11(22.9)$ & $37(77.1)$ & 0.907 \\
\hline Business & $8(23.5)$ & $26(76.5)$ & \\
\hline Labour & $2(14.3)$ & $12(85.7)$ & \\
\hline \multicolumn{4}{|l|}{$\begin{array}{l}\text { Occupation of } \\
\text { respondents' husband }\end{array}$} \\
\hline Service & $26(32.9)$ & $53(67.1)$ & \\
\hline Business & $18(21.7)$ & $65(78.3)$ & \\
\hline Labour & $9(16.7)$ & $45(83.3)$ & 0.042 \\
\hline Foreign employment & $16(16.3)$ & $82(83.7)$ & \\
\hline \multicolumn{4}{|l|}{$\begin{array}{l}\text { Annual family income } \\
\text { (in NPR) }\end{array}$} \\
\hline Less than 250000 & $24(20.5)$ & $93(79.5)$ & \\
\hline 250000 to 600000 & $35(22.2)$ & $123(77.8)$ & 0.797 \\
\hline More than 600000 & $10(25.6)$ & $29(74.4)$ & \\
\hline \multicolumn{4}{|l|}{ Parity } \\
\hline One & $36(23.2)$ & $119(76.8)$ & 0.597 \\
\hline More than one & $33(20.8)$ & $126(79.2)$ & \\
\hline \multicolumn{4}{|l|}{ Number of ANC visit } \\
\hline$<4$ times & $4(11.1)$ & $32(28.1)$ & 0.101 \\
\hline$\geq 4$ times & $65(23.4)$ & $213(76.6)$ & \\
\hline \multicolumn{4}{|l|}{$\begin{array}{l}\text { Type of facility used for } \\
\text { delivery }\end{array}$} \\
\hline Government hospital & $38(14.3)$ & $227(85.7)$ & $<0.001$ \\
\hline Private hospital & $31(63.3)$ & $18(36.7)$ & \\
\hline \multicolumn{4}{|l|}{$\begin{array}{l}\text { Had complication } \\
\text { during childbirth }\end{array}$} \\
\hline Yes & $19(31.7)$ & $41(68.3)$ & 0.044 \\
\hline No & $50(22.6)$ & $204(80.3)$ & \\
\hline \multicolumn{4}{|l|}{$\begin{array}{l}\text { Awareness level on PNC } \\
\text { services }\end{array}$} \\
\hline Poor & $27(17.2)$ & $130(82.8)$ & 0.041 \\
\hline Good & $42(26.8)$ & $115(73.2)$ & \\
\hline
\end{tabular}


TABLE 5: Odds ratio of factors associated with use of complete PNC.

\begin{tabular}{|c|c|c|c|}
\hline Variables & Unadjusted OR (95\% CI) & Adjusted OR (95\% CI) & Adjusted $p$ value \\
\hline \multicolumn{4}{|c|}{ Occupation of respondents' husbands } \\
\hline Service (job holder) & $2.51(1.23-5.12)$ & $3.49(1.50-8.13)$ & 0.004 \\
\hline Business & $1.41(0.67-2.99)$ & $1.69(0.71-4.00)$ & 0.229 \\
\hline Labour & $1.02(0.41-2.50)$ & $2.11(0.72-6.18)$ & 0.171 \\
\hline Foreign employment & 1 & 1 & \\
\hline \multicolumn{4}{|c|}{ Type of facility used for delivery } \\
\hline Government hospital & 1 & 1 & \\
\hline Private hospital & $10.2(5.23-20.2)$ & $11.4(5.40-24.2)$ & $<0.001$ \\
\hline \multicolumn{4}{|l|}{ Caste/ethnicity } \\
\hline Janajati & $2.71(1.13-6.48)$ & $2.87(1.09-7.53)$ & 0.031 \\
\hline Brahman/Chhetri & $2.87(1.26-6.54)$ & $3.18(1.24-8.12)$ & 0.016 \\
\hline Dalits & 1 & 1 & \\
\hline \multicolumn{4}{|l|}{ Awareness level on PNC } \\
\hline Poor & 1 & 1 & \\
\hline Good & $1.75(1.02-3.03)$ & $1.34(0.71-2.54)$ & 0.362 \\
\hline \multicolumn{4}{|c|}{ Complication during childbirth } \\
\hline Yes & $1.89(1.01-3.53)$ & $1.25(0.58-2.67)$ & 0.561 \\
\hline No & 1 & 1 & \\
\hline
\end{tabular}

1 indicates reference category.

husbands were not involved in agriculture but were in foreign employment along with in business, labour, and service. This could be because of the urban setting of the study. Involvement of male is necessary for improved maternal health outcomes in developing countries [33]. Husbands have an important role in the maternity care of their wives [34]. A study conducted in Myanmar revealed that male involvement is more likely (aOR, 2.19; 95\% CI, and 1.02-4.69) to result in having complete PNC [29]. In Nepal, husbands' involvement in maternal health care is in giving advice, supporting with household work, and arranging money and transportation for the delivery in a health facility [34]. In addition, the husbands may be the one to accompany wives to health facilities [35]. A husband accompanying his wife is associated with higher likelihood of using PNC from a trained service provider [36]. The involvement of the husband is influenced by his availability, cultural beliefs, and traditions [35].

The present study depicted that respondents who delivered their last child at a private hospital were more likely $(\mathrm{aOR}=11.9,95 \% \mathrm{CI}: 5.52-25.7)$ to have complete PNC as compared to those who delivered in a government hospital. This finding is supported by the study conducted in Palestine $(\mathrm{aOR}=1.8,95 \% \mathrm{CI}: 1.0-3.4)$ that women who delivered at a private hospital had a higher likelihood to have PNC [37]. Consistent results were observed in a study in Zambia where women giving birth at a private hospital/clinic had higher odds (aOR 10.08 95\% CI 3.35-30.35) of having PNC than at a government hospital (aOR 7.24, 95\% CI 4.92-11.84) or government health center/clinic (aOR 7.15 95\% CI 4.7910.66) [38]. PNC counseling and provision of appointment are associated with a higher likelihood of using PNC service [28]. Timeliness and hospitality to patients are greater in private facilities than in public [39]. Women preferred private facilities for maternal health services because of longer consultation time, better interpersonal and communication skills of health care providers, better treatment, more advanced equipment and devices, availability of female obstetricians, and more flexible appointment times [40]. Women who delivered at health facilities rated private facilities to be of higher quality in terms of amenities and interpersonal aspects than public ones [41]. Consistently, interpersonal process was also found of better quality in private health facilities by the women attending health facilities for family planning service [42]. Public facilities are generally used due to financial and physical accessibility [43].

\section{Strengths and Limitations of the Study}

There was a possibility of recall bias as the mothers were asked about the utilization of the service received within last one year prior to the data collection. The findings may not be generalizable in all places as the sample was restricted to four wards of the municipality. Some risk factors have not been measured in this study such as neonatal complication and sociocultural practices during puerperium. However, the study has some strengths as well. The study identifies the status of the utilization of complete PNC in which very limited information is available in community-based studies in Nepal. Secondly, the study shows the status of the utilization of PNC in an urban setting where almost all mothers had institutional delivery, unlike in previous studies of Nepal.

\section{Conclusion}

The utilization of complete PNC is low, although having at least one PNC was high, despite the availability of and 
accessibility to the service. Caste/ethnicity, occupation of respondents' husband, and type of health facility used for delivery were found to have influenced the utilization. Uptake of complete PNC service may increase by raising awareness on the importance and schedule of PNC. PNC services should be focused on the mothers who belong to a disadvantaged caste/ethnicity and whose husbands are in foreign employment. Involvement of husbands in maternity care, including in PNC service utilization, should be promoted. In addition, improving the quality of the service, particularly in government health facilities, such as interpersonal aspects, timely service, and amenities, may increase the use of PNC service. Implementation research to explore feasibility and sustainability of providing complete PNC by a health worker such as ANM by home visits is needed considering difficult geography and fragile condition of the mother and newborn to travel to a health facility for second and third PNC.

\section{Abbreviations \\ ANC: Antenatal care \\ ANM: Auxiliary nurse midwife \\ aOR: Adjusted odds ratio \\ CI: Confidence interval \\ NPR: Nepalese rupees \\ PNC: Postnatal care.}

\section{Data Availability}

Data will be available from the corresponding author on reasonable request.

\section{Ethical Approval}

Ethical approval was obtained from Chitwan Medical College-Institutional Review Committee.

\section{Consent}

Informed verbal consent was obtained from each respondent. Confidentiality of information and privacy were maintained.

\section{Conflicts of Interest}

The authors declare that they have no competing interests.

\section{Authors' Contributions}

SC conceptualized the design of the study, prepared tools, collected and analyzed data, interpreted results, and drafted the manuscript. RS contributed to the conceptualization and design of the analysis, interpreted data, reviewed literature, and contributed extensively to the drafting of the manuscript. LR supported in the conceptualization of the study, data analysis and interpretation, and provided feedback throughout the research. All authors have read and approved the final version of the manuscript.

\section{Acknowledgments}

The authors would like to thank the faculty of nursing department, Chitwan Medical College, as well as the Baglung municipality for their support during the study period.

\section{References}

[1] World Health Organization, UNICEF, Trends in maternal mortality: 1990-2015: estimates from WHO, UNICEF, UNFPA, World Bank Group and the United Nations Population Division, World Health Organization, 2015.

[2] E. Sines, U. Syed, S. Wall, and H. Worley, "Postnatal care: a critical opportunity to save mothers and newborns," in Policy Perspectives on Newborn Health, pp. 1-7, Population Reference Bureau, 2007.

[3] World Health Organization, WHO recommendations on postnatal care of the mother and newborn, World Health Organization, 2014.

[4] Ministry of Health, New ERA, ORC Macro, Nepal demographicand health survey 2016, Family health division, Ministry of Health; New ERA; ORC Macro, Calverton, Maryland, 2017.

[5] National Planning Commission, "Sustainable development goals, 2016-2030, National (preliminary) report,” Nepal Government of Nepal, National Planning Commission, Kathmandu, 2015.

[6] World Health Organization, WHO technical consultation on postpartum and postnatal care, World Health Organization, Geneva, 2010.

[7] Ministry of Health and Population, National medical standard for reproductive health. Volume III: maternal and neonatal care, Ministry of health and population, Family health division, Nepal, 2009.

[8] Department of Health Services, Annual report 2015/2016, Department of Health Services, Ministry of Health and Population, Government of Nepal, Kathmandu, Nepal, 2017.

[9] Department of Health Services, Annual report 2017/2018, Department of Health Services, Ministry of Health and Population, Government of Nepal, Kathmandu, Nepal, 2019.

[10] A. Singh, S. S. Padmadas, U. S. Mishra, S. Pallikadavath, F. A. Johnson, and Z. Matthews, "Socio-economic inequalities in the use of postnatal care in India," PLoS One, vol. 7, no. 5, article e37037, 2012.

[11] S. Dhakal, G. N. Chapman, P. P. Simkhada, E. R. Van Teijlingen, J. Stephens, and A. E. Raja, "Utilisation of postnatal care among rural women in Nepal," BMC Pregnancy and Childbirth, vol. 7, no. 1, p. 19, 2007.

[12] R. Adhikari, "Effect of Women's autonomy on maternal health service utilization in Nepal: a cross sectional study," BMC Women's Health, vol. 16, no. 1, p. 26, 2016.

[13] M. Paudel, V. Khanal, B. Acharya, and M. Adhikari, "Determinants of postnatal service utilization in a Western District of Nepal: community based Cross Sectional study," Journal of Womens Health Care, vol. 2, no. 3, p. 2167, 2013.

[14] V. Khanal, M. Adhikari, R. Karkee, and T. Gavidia, "Factors associated with the utilisation of postnatal care services among the mothers of Nepal: analysis of Nepal demographic and health survey 2011," BMC Women's Health, vol. 14, no. 1, p. 19, 2014.

[15] District Public Health Office, District population profile 2017, Nepal, DPHO, Baglung, 2017. 
[16] L. Naing, T. Winn, and B. Rusli, "Practical issues in calculating the sample size for prevalence studies," Archives of Orofacial Sciences, vol. 1, pp. 9-14, 2006.

[17] R. Arya, B. Antonisamy, and S. Kumar, "Sample size estimation in prevalence studies," The Indian Journal of Pediatrics, vol. 79, no. 11, pp. 1482-1488, 2012.

[18] E. van Teijlingen and V. Hundley, "Pilot studies in family planning and reproductive health care," Journal of Family Planning and Reproductive Health Care, vol. 31, no. 3, pp. 219-221, 2005.

[19] J. P. Pandey, M. R. Dhakal, S. Karki, P. Poudel, and M. S. Pradhan, Maternal and Child Health in Nepal: The Effects of Caste, Ethnicity, and Regional Identity:Further Analysis of the 2011 Nepal Demographic and Health Survey.Kathmandu: Calverton, Nepal Ministry of Health and Population, New ERA, and ICF International, Maryland, USA, 2013.

[20] National Institute for Research and Training, Nepal education sector analysis, National Institute for Research and Training (NIRT), Kathmandu, Nepal, 2017, Vii..

[21] A. A. Rurangirwa, I. Mogren, L. Nyirazinyoye, J. Ntaganira, and G. Krantz, "Determinants of poor utilization of antenatal care services among recently delivered women in Rwanda; a population-based study," BMC Pregnancy and Childbirth, vol. 17, no. 1, p. 142, 2017.

[22] S. Pandey and S. Karki, "Socio-economic and demographic determinants of antenatal care services utilization in Central Nepal," International Journal of MCH and AIDS, vol. 2, no. 2, pp. 212-219, 2014.

[23] O. M. Campbell, L. Cegolon, D. Macleod, and L. Benova, "Length of stay after childbirth in 92 countries and associated factors in 30 Low- and middle-income countries: compilation of reported data and a cross-sectional analysis from nationally representative surveys," PLoS Medicine, vol. 13, no. 3, article e1001972, 2016.

[24] J. M. Lauristen and M. Bruus, Epi Tour: An Introduction to Validated Data Entry and Documentation of Data by Use of EpiData, The Epidata Association, Odense Denmark, 2008.

[25] M. Norusis, SPSS 16.0 Statistical Procedures Companion, Prentice Hall Press, 2008.

[26] C.-Y. J. Peng, K. L. Lee, and G. M. Ingersoll, "An introduction to logistic Regression analysis and reporting," The Journal of Educational Research, vol. 96, no. 1, pp. 3-14, 2010.

[27] A. Sharma, P. S. Thakur, P. K. Kasar, R. Tiwari, and R. Sharma, "Utilization of post natal care in tribal area of Madhya Pradesh, India: a community based cross-sectional study," International Journal of Medical Science and Public Health., vol. 3, no. 10, pp. 1266-1272, 2014.

[28] S. Berhanu Sr., Y. Asefa, and B. W. Giru, "Prevalence of postnatal care utilization and associated factors among women who gave birth and attending immunization clinic in selected government health centers in Addis Ababa, Ethiopia, 2016," Prevalence, vol. 26, 2016.

[29] A. S. Mon, M. K. Phyu, W. Thinkhamrop, and B. Thinkhamrop, Utilization of full postnatal care services among rural Myanmar women and its determinants: a crosssectional study, F1000Research, 2018.

[30] É. V. Langlois, M. Miszkurka, M. V. Zunzunegui, A. Ghaffar, D. Ziegler, and I. Karp, "Inequities in postnatal care in lowand middle-income countries: a systematic review and metaanalysis," Bulletin of the World Health Organization, vol. 93, no. 4, pp. 259-270, 2015.
[31] L. Bennett, "Gender, caste and ethnic exclusion in Nepal," in Following the policy process from analysis to action, World Bank, Washington, DC, 2005.

[32] R. Khadka, S. A. Hong, and B. O. Thepthien, "Postnatal care service utilization and its determinants in Ramechhap district, Nepal: a community-based cross-sectional study," Journal of Public Health and Development, vol. 15, no. 3, pp. 1-6, 2017.

[33] J. Yargawa and J. Leonardi-Bee, "Male involvement and maternal health outcomes: systematic review and meta-analysis," Journal of Epidemiology and Community Health, vol. 69, no. 6, pp. 604-612, 2015.

[34] D. K. Thapa and A. Niehof, "Women's autonomy and husbands' involvement in maternal health care in Nepal," Social Science \& Medicine, vol. 93, pp. 1-10, 2013.

[35] S. Lewis, A. Lee, and P. Simkhada, "The role of husbands in maternal health and safe childbirth in rural Nepal: a qualitative study," BMC Pregnancy and Childbirth, vol. 15, no. 1, p. 162, 2015.

[36] A. E. Rahman, J. Perkins, S. Islam et al., "Knowledge and involvement of husbands in maternal and newborn health in rural Bangladesh," BMC Pregnancy and Childbirth, vol. 18, no. 1, p. 247, 2018.

[37] E. Dhaher, R. T. Mikolajczyk, A. E. Maxwell, and A. Krämer, "Factors associated with lack of postnatal care among Palestinian women: a cross-sectional study of three clinics in the West Bank," BMC Pregnancy and Childbirth, vol. 8, no. 1, p. 26, 2008.

[38] C. Chungu, M. Makasa, M. Chola, and C. N. Jacobs, "Place of delivery associated with postnatal care utilization among childbearing women in Zambia," Frontiers in Public Health, vol. 6, p. 94, 2018.

[39] S. Basu, J. Andrews, S. Kishore, R. Panjabi, and D. Stuckler, "Comparative performance of private and public healthcare systems in low-and middle-income countries: a systematic review," PLoS Medicine, vol. 9, no. 6, article e1001244, 2012.

[40] M. S. Alyahya, Y. S. Khader, A. Batieha, and M. Asad, "The quality of maternal-fetal and newborn care services in Jordan: a qualitative focus group study," BMC Health Services Research, vol. 19, no. 1, p. 425, 2019.

[41] R. Karkee, A. H. Lee, and P. K. Pokharel, "Women's perception of quality of maternity services: a longitudinal survey in Nepal," BMC Pregnancy and Childbirth, vol. 14, no. 1, p. 45, 2014.

[42] P. L. Hutchinson, M. Do, and S. Agha, "Measuring client satisfaction and the quality of family planning services: a comparative analysis of public and private health facilities in Tanzania, Kenya and Ghana," BMC Health Services Research, vol. 11, no. 1, p. 203, 2011.

[43] R. Karkee and J. Kadariya, "Choice of health-care facility after introduction of free essential health services in Nepal," WHO South-East Asia Journal of Public Health, vol. 2, no. 2, pp. 96-100, 2013. 